The Bulletin of Symbolic Logic

Volume 10, Number 3, Sept. 2004

\title{
TRANSFINITE PROGRESSIONS: A SECOND LOOK AT COMPLETENESS
}

\author{
TORKEL FRANZÉN
}

§1. Iterated Gödelian extensions of theories. The idea of iterating ad infinitum the operation of extending a theory $T$ by adding as a new axiom a Gödel sentence for $T$, or equivalently a formalization of " $T$ is consistent", thus obtaining an infinite sequence of theories, arose naturally when Gödel's incompleteness theorem first appeared, and occurs today to many non-specialists when they ponder the theorem. In the logical literature this idea has been thoroughly explored through two main approaches. One is that initiated by Turing in his "ordinal logics" (see Gandy and Yates [2001]) and taken very much further in Feferman's work on transfinite progressions, which also introduced the more general study of extensions by reflection principles, of which consistency statements are a special case. This approach starts from an assignment of theories to ordinal notations, and extracts sequences of theories through a suitable choice of a path in the set of ordinal notations. The second approach, illustrated in particular by the work of Schmerl and Beklemishev, starts instead from a suitably well-behaved primitive recursive well-ordering, which is used to define a sequence of theories. This second approach has led to precise results about the relative proof-theoretical strength of sequences of theories obtained by iterating different reflection principles. The Turing-Feferman approach, on the other hand, lends itself well to an investigation in qualitative and philosophical terms of the relevance of such iterated reflection extensions to mathematical knowledge, in particular because of two developments associated with this approach. First, there is Feferman's famous completeness theorem for transfinite progressions based on full (uniform) reflection, which exercises a powerful appeal on the imagination, but which (perhaps because of the somewhat inaccessible character of Feferman [1962b]) is not widely known in any detail. Second, there is the concept of the autonomous part of a progression, introduced by Kreisel and Feferman, which allows us to reason in qualitative terms about what it is we do know, potentially at least, on the basis of iterated reflection principles.

Received January 26, 2004; accepted April 2, 2004. 
Autonomy plays a large role in Franzén [2004], which is concerned with the peculiar role of reflection principles in demonstrating the apparent inexhaustibility of our mathematical knowledge. The topic of the present paper is the completeness theorem for progressions, which was only touched on briefly in Franzén [2004]. The purpose of the presentation is twofold. First, to give an updated version of Feferman's completeness result partly based on later developments, aiming to clarify the argument rather than include every detail. Second, to answer the natural non-technical question just what it is about reflection principles that makes it possible to prove, by iterating such principles, any true arithmetical sentence, and just where and how such proofs leave our actual or potential mathematical knowledge behind. Some open questions which to the best of my knowledge have not been settled in the literature will also be formulated. Hopefully the presentation will be useful to philosophers and logicians who are not already familiar with the topic and wish to understand what the completeness theorem for progressions is all about.

Feferman's completeness proof has two main ingredients. The first is the use of convoluted non-standard definitions of the axioms of a theory, by which iterated reflection principles can be formulated in such a way as to imply any given true $\Pi_{2}$-statement of arithmetic. The second is the use of sequences of theories to mimic the application of an infinitary rule of inference known to be complete for arithmetical sentences through work by Shoenfield. The logical prerequisites for a discussion of these matters will be summarized in the next section.

§2. Logical preliminaries. The discussion will be restricted to effectively axiomatizable first order extensions of PA, so by a theory will be meant such an extension, unless otherwise noted. We will need to use the arithmetical formula hierarchy, partial truth definitions, arithmetical reflection principles, the recursion theorem, the basic facts about Kleene's system $\mathbf{O}$ of ordinal notations, and a few facts about sequence numbers and trees. This material will be summarized here, and the notation of the paper explained. For proofs and further details, see e.g., Shoenfield [1967], Franzén [2004], Hájek and Pudlák [1993].

The arithmetical formula hierarchy. An arithmetical formula will be taken to be one written in the language of PA extended with a symbol $<$ for the ordering relation between natural numbers. The numerals are the terms $0, s(0), s(s(0)), \ldots$, and we write $\bar{n}$ for the numeral denoting $n$. Using logical and arithmetical equivalences, any formula $\phi$ in this language can be transformed into a $\Delta_{0}$-formula, a $\Sigma_{n}$-formula or a $\Pi_{n}$-formula which is equivalent in PA to $\phi$.

In $\Delta_{0}$-formulas all quantifiers are bounded:

$$
\forall x(x<t \supset \phi) \text {, written } \forall x<t \phi,
$$




$$
\exists x(x<t \wedge \phi) \text {, written } \exists x<t \phi
$$

where in both cases $x$ does not occur in $t$.

$\Sigma_{n}$-formulas, for $n>0$, are prenex formulas with alternating quantifiers $\exists$ and $\forall$

$$
Q_{1} x_{1} \ldots Q_{n} x_{n} \psi
$$

where $Q_{1}$ is $\exists$ and $\psi$ is a $\Delta_{0}$-formula. In a $\Pi_{n}$-formula, $Q_{1}$ is $\forall$. By a $\Pi_{0}$-formula or a $\Sigma_{0}$-formula is meant a $\Delta_{0}$-formula.

Disjunctions and conjunctions of $\Pi_{n}$-formulas are equivalent in PA to $\Pi_{n}$-formulas, and similarly for $\Sigma_{n}$-formulas.

Theories and theorems. $\Sigma_{1}$-formulas will play a special role in the following, because a $\Sigma_{1}$-formula with $n$ free variables defines an $n$-ary effectively enumerable relation, and conversely every such relation is definable by a $\Sigma_{1}$-formula. In particular, an effectively axiomatizable theory $T$ is given by a $\Sigma_{1}$-formula $\phi$ defining the axioms of $T$. Given $\phi, \operatorname{Thm}_{\phi}(x)$ is defined as a formalization in arithmetic of " $x$ is (the Gödel number of) a formula derivable using the rules of predicate logic from formulas satisfying $\phi$ ". This can also be written $\operatorname{Thm}_{T}(x)$ when the formula $\phi$ is implicit, but it is a pervasive aspect of the subject that $\operatorname{Thm}_{\phi}(x)$ and $\operatorname{Thm}_{\psi}(x)$ are not in general provably equivalent (in the theories considered) even when $\phi$ and $\psi$ define the same set of axioms.

The parenthetical "the Gödel number of" will be left out in what follows, where any of the standard codings of formulas, proofs, and other syntactic objects as natural numbers may be assumed used.

The set of derivations from formulas satisfying $\phi$ is not in general recursive. We therefore define a primitive recursive relation $\operatorname{Prf}_{\phi}(u, y)$ using (a variant of) Craig's construction. If $\phi$ is $\exists y \psi(x, y), \operatorname{Prf}_{\phi}(u, y)$ holds if and only if $y$ is a derivation of $u$ from formulas satisfying

$\exists w<x \exists y<x(\psi(w, y) \wedge x$ is the conjunction of $w$ with

$$
\bar{n}=\bar{n} \text { for some } n<x) \text {. }
$$

On this definition, $\operatorname{Prf}_{\phi}$ is a primitive recursive relation, and it is provable in PA that $\operatorname{Thm}_{\phi}(u)$ holds if and only there is a $y$ such that $\operatorname{Prf}_{\phi}(u, y)$. We say that $y$ is a $P R$-proof of $u$ if $\operatorname{Prf}_{\phi}(u, y)$ holds.

By the $\Sigma_{1}$-completeness theorem, every true $\Sigma_{1}$-sentence is provable in PA (and in fact in Robinson arithmetic, a weak subtheory of PA).

Partial truth definitions. For every $n$, we can define an arithmetical formula True- $\Sigma_{n}(x)$ expressing that $x$ is a true $\Sigma_{n}$-sentence, and similarly $\operatorname{True}-\Pi_{n}(x)$. For $n>0$, True- $\Sigma_{n}(x)$ is itself a $\Sigma_{n}$-formula and $\operatorname{True}-\Pi_{n}(x)$ a $\Pi_{n}$-formula. For any $\Sigma_{n}$-formula $\phi\left(x_{1}, \ldots, x_{m}\right)$ with the indicated free variables (and similarly for $\Pi_{n}$-formulas), PA proves a formalization of 
For every $y_{1}, \ldots, y_{m}$, the formula obtained by substituting the numeral for $y_{i}$ for the variable $x_{i}$ in $\phi$ (for $i=1, \ldots, m$ ) is a true $\Sigma_{n}$-sentence if and only if $\phi\left(y_{1}, \ldots, y_{m}\right)$.

PA also proves, for any $n>0$, a formalization of

For any $\phi, \exists x \phi(x)$ is a true $\Sigma_{n}$-sentence if and only if $\phi(\bar{k})$ is a true $\Pi_{n-1}$-sentence for some $k$

and similarly for $\Pi_{n}$-sentences.

Using such restricted definitions of semantic concepts, PA proves e.g., that $\operatorname{Thm}_{\phi}(u)$ holds if and only there is a $y$ such that $\operatorname{Prf}_{\phi}(u, y)$, not only for each fixed formula $\phi$, but as a property of $\Sigma_{1}$-formulas generally: for every $\phi$ and every $u, \operatorname{Thm}_{\phi}(u)$ if and only there is a $y$ such that $\operatorname{Prf}_{\phi}(u, y)$.

A theory $T$ is sound if every sentence provable in $T$ is true, and $\Sigma_{n}$-sound if every $\Sigma_{n}$-sentence provable in $T$ is true. $\Pi_{n}$-soundness is defined similarly. Thus $\Sigma_{0}$-soundness is equivalent to consistency. " $T$ is $\Sigma_{n}$-sound" is (equivalent in PA to) a $\Pi_{n+1}$-formula.

Reflection principles. A reflection principle for a theory $T$ is, informally, a statement about $T$ that is a consequence of $T$ being sound. Thus the most obvious reflection principle for $T$ is the assertion that $T$ is sound. The completeness theorems for progressions, however, deal with reflection principles that can be formulated in the language of arithmetic. For this, we use a partial truth definition to formulate the weaker reflection principle that $T$ is $\Sigma_{n}$-sound.

For every $n, \operatorname{REF}^{n}(\phi)$, where $\phi$ is a $\Sigma_{1}$-formula defining the axioms of a theory $T$, is an arithmetical $\Pi_{n+1}$-sentence formalizing " $T$ is $\Sigma_{n}$-sound". $\operatorname{REF}^{n}(\phi)$ is equivalent in PA to " $T$ is $\Pi_{n+1}$-sound", by a formalization of the following argument: if $\forall x \exists y \phi(x, y)$ is a $\Pi_{n+1}$-theorem of $T, \exists y \phi(\bar{k}, y)$ is a $\Sigma_{n}$-theorem of $T$ for every $k$, and hence is $\Sigma_{n}$-true, so $\forall x \exists y \phi(x, y)$ is $\Pi_{n+1}$-true.

We define an extension by $n$-reflection of a theory $T$ as a theory $T+$ $\operatorname{REF}^{n}(\phi)$ where $\phi$ is some $\Sigma_{1}$-formula defining the axioms of $T$, in the extensional sense that the axioms of $T$ are the formulas satisfying $\phi$. For uniformity of notation, we let $\operatorname{REF}^{\omega}(\phi)$ stand for the collection of all $\operatorname{REF}^{n}(\phi)$, so that $T+\operatorname{REF}^{\omega}(\phi)$ is what is usually called an extension by (full) uniform reflection of $T$, with every $\operatorname{REF}^{n}(\phi)$ as a new axiom. A 0 -reflection extension will also be referred to as a consistency extension.

In general, $\operatorname{REF}^{n}(\phi)$ and $\operatorname{REF}^{n}(\psi)$ are not equivalent in $T$ for different choices of $\Sigma_{1}$-formulas $\phi$ and $\psi$ defining the axioms of $T$. When we are talking about theories in general, there is no more specific condition to impose on the formula $\phi$, and no basis for distinguishing between different choices of $\phi$. By Feferman's work in Feferman [1960], $\phi$ being a $\Sigma_{1}$-formula is enough to ensure that any extension by reflection of $T$ is logically stronger than $T$. However, an extension by $n+1$-reflection is not in general stronger 
than an extension by $n$-reflection, unless the same formula is used to define the axioms of $T$ in both extensions. (This is a consequence of the fact, which will emerge below, that definitions $\phi$ and $\psi$ of the axioms of $T$ can be chosen so that $T+\operatorname{REF}^{0}(\phi)$ proves the consistency of $T+\operatorname{REF}^{n}(\psi)$.)

In the case of theories which we actually use to formalize part of our mathematical knowledge - theories like PA and ZFC - and for various extensions and subtheories of such theories, there is a canonical definition of their axioms, of the form "an axiom of $T$ is one of the formulas $\phi_{1}, \ldots, \phi_{n}$ or an instance of one of the schemata $\Phi_{1}, \ldots, \Phi_{m}$ ", which then yields corresponding canonical reflection principles. There are also canonical definitions of the axioms of various theories which do not have this form, as when we add an infinity of iterated consistency statements to the axioms of PA, but characterizing such canonical definitions is a problematic matter. A definition of the axioms of one of these theories which is not equivalent in PA to the canonical definition will be called non-standard. As will be seen, the reflection-specific part of Feferman's completeness proof depends on using somewhat opaque non-standard definitions of the axioms of a theory.

The system $\mathbf{O}$. The notations in $\mathbf{O}$ are defined by a simultaneous induction together with the partial ordering $<_{\mathbf{O}}$ of notations and the ordinal $|a|$ of a notation $a$, where we use Kleene's notation $\{e\}$ for the unary partial recursive function with index $e . \operatorname{suc}(a)$ and $\lim (e)$ can be taken to be e.g., $2^{a}$ and $3^{e}$.

0 is in $\mathbf{O}$, and $|0|=0$.

If $a$ is in $\mathbf{O}$ then $\operatorname{suc}(a)$ is in $\mathbf{O}, a<_{\mathbf{O}} \operatorname{suc}(a)$ and $|\operatorname{suc}(a)|=|a|+1$.

If $e$ is the index of a total function and $\{e\}(n)<_{\mathbf{0}}\{e\}(n+1)$ for every $n$, then $\lim (e)$ is in $\mathbf{O},\{e\}(n)<_{\mathbf{O}} \lim (e)$ for every $n$, and $|\lim (e)|$ is the supremum of the ordinals $|\{e\}(n)|$ for $n=0,1, \ldots$

If $a<_{\mathbf{O}} b$ and $b<_{\mathbf{O}} c$ then $a<_{\mathbf{O}} c$.

$a<_{\mathbf{O}} b$ implies $|a|<|b|$, although the converse does not hold, so $<_{\mathbf{O}}$ is a well-founded relation. $\left\{b \mid b<_{\mathbf{O}} a\right\}$ is a special case of a path in $\mathbf{O}$, that is, a totally ordered subset which is closed under predecessors. The length of a path $P$ is the ordinal of the restriction of $<_{\mathbf{0}}$ to $P$, so that the length of $\left\{b \mid b<_{\mathbf{O}} a\right\}$ is $|a|$. The numbers in a path $P$ give a unique notation to every ordinal smaller than the length of $P$.

The relation $<_{\mathbf{O}}$ is not effectively enumerable, but for every $a$ in $\mathbf{O}$, the restriction of $<_{\mathbf{O}}$ to $\left\{b \mid b<_{\mathbf{O}} a\right\}$ is effectively enumerable, and coincides on this set with the effectively enumerable relation $<_{\mathbf{K}}$ inductively defined by

$$
\begin{aligned}
& a<_{\mathbf{K}} \operatorname{suc}(a) \text { for every } a, \\
& \{e\}(n)<_{\mathbf{K}} \lim (e) \text { for every } n \text { such that }\{e\}(n) \text { is defined, } \\
& \text { if } a<_{\mathbf{K}} b \text { and } b<_{\mathbf{K}} c \text { then } a<_{\mathbf{K}} c .
\end{aligned}
$$

The natural numbers $0,1,2, \ldots$ have unique notations

$$
0, \operatorname{suc}(0), \operatorname{suc}(\operatorname{suc}(0)), \ldots
$$


in $\mathbf{O}$, and we write $n_{\mathbf{O}}$ for the notation in $\mathbf{O}$ denoting $n$.

The recursion theorem. We will use Kleene's recursion theorem in the form

For any primitive recursive $F$ there is an $e$ such that $\{e\}$ is primitive recursive, and provably in PA

$$
\{e\}(k)=F(e, k) \text { for every } \mathrm{k} .
$$

We will also use the following variant:

For any primitive recursive $G, H, J$ there is a primitive recursive $F$ such that it is provable in PA that for all $a, e, k$

$F(0, k)=G(k)$,

$F(\operatorname{suc}(a), k)=H(a, k, F(a, k))$,

$F(\lim (e), k)=J(e, b, k)$, where $\{b\}(m)=F(\{e\}(m), k)$ for

every $m$ for which $\{e\}(m)$ is defined and $b$ is given as a primitive recursive function of $e, k$.

As an application of the latter formulation we can define $a+b$ so that (provably in PA) $a+0=0, a+\operatorname{suc}(b)=\operatorname{suc}(a+b)$, and $a+\lim (e)=\lim (f)$ where $\phi(n)=a+\{e\}(n)$ for every $n$ for which $\{e\}(n)$ is defined. An inductive proof shows that for $a, b$ in $\mathbf{O},|a+b|=|a|+|b|$, and if $b$ is not $0, a<_{\mathbf{O}} a+b$. (Since we never apply ordinary arithmetical addition to notations, the use of + for this operation will not cause any confusion.)

Sequence numbers. We presuppose in the following some primitive recursive coding of sequences of natural numbers as numbers, writing $\left\langle a_{1}, \ldots, a_{k}\right\rangle$ both for a sequence of numbers and for the corresponding sequence number. \langle\rangle is the empty sequence. The set $S e q$ of sequence numbers is primitive recursive, as is the function length $(s)$ giving the length of $s$ and also the function $(s)_{i}$ giving the $i$-th element in $s$, counting the first element as the 0 -th. (If $s$ is not a sequence number or $i$ is greater than or equal to the length of $s$, the value of these functions is 0 .) The primitive recursive operation of concatenation of sequence numbers is defined so that $\left\langle a_{1}, \ldots, a_{k}\right\rangle *\left\langle b_{1}, \ldots, b_{m}\right\rangle=\left\langle a_{1}, \ldots, a_{k}, b_{1}, \ldots, b_{m}\right\rangle$.

A partial ordering of sequence numbers is defined by $s<s^{\prime}$ iff $s^{\prime}=s * s^{\prime \prime}$ for some non-empty $s^{\prime \prime}$. Seq with this partial ordering is the full $\omega$-tree, and a tree of sequence numbers is a subset $S$ of $S e q$ which is closed under predecessors. When a strict partial order $R$ is spoken of as a tree of sequence numbers, this means that $R$ is the restriction of $<$ to a tree of sequence numbers. $S$ is well-founded if it has no infinite path, which is the same as saying that the converse $>_{S}$ of the relation $<$ restricted to $S$ is a well-founded relation. The ordinal $\operatorname{ord}_{S}(a)$ of $a$ in $S$ is defined by recursion on this wellfounded relation as the supremum of $\operatorname{ord}_{S}(a *\langle n\rangle)+1$ for the $n$ such that $a *\langle n\rangle$ is in $S$, and the ordinal $|S|$ of $S$ is the ordinal of \langle\rangle .

The operations on sequences are defined so that $(s)_{i}$ is strictly smaller than $s$, and $s$ strictly smaller than $s * s^{\prime}$ for non-empty $s^{\prime}$, so that we can 
define functions on sequence numbers by primitive recursion in terms of their elements and proper subsequences.

§3. Reflection sequences and progressions. An iterated $n$-reflection extension of a theory $T_{0}$ is naturally thought of as a theory in a sequence

$$
T_{0}, T_{1}, \ldots, T_{\omega}, T_{\omega+1}, \ldots, T_{\alpha}, \ldots
$$

of theories, where $T_{\alpha+1}$ is an extension by $n$-reflection of $T_{\alpha}$ and $T_{\lambda}$ for limit ordinals $\lambda$ has as axioms the union of the axioms of earlier theories. There is no apparent reason why there should not be such a sequence for any initial segment of the countable ordinals. However, if the formulas $\operatorname{REF}^{n}(\phi)$ are to be interpretable as expressing iterated reflection, the definitions $\phi$ must be formulated in terms of arithmetically definable well-orderings, or equivalently in terms of effectively enumerable well-orderings, which we may assume to be given by notations in $\mathbf{O}$. We therefore take as basic the definition of an $n$-reflection sequence as a mapping taking notations $a$ in some path $P$ in $\mathbf{O}$ to $\Sigma_{1}$-formulas $\phi_{a}$, such that, with $T_{a}=$ the theory with axioms defined by $\phi_{a}$, the following two characteristic conditions are satisfied:

$$
\begin{aligned}
T_{\text {suc }(a)} & =T_{a}+\operatorname{REF}^{n}\left(\phi_{a}\right), \\
T_{\lim (e)} & =\bigcup_{n} T_{\{e\}(n)} .
\end{aligned}
$$

We accordingly define an iterated $n$-reflection extension of $T_{0}$ (where we include the case $n=\omega$ ) as a theory that occurs in some such sequence. Note that if $T$ is $\Sigma_{n+1}$-sound, so is every iterated $n$-reflection extension of $T$, by the following argument. $\operatorname{REF}^{n}\left(\phi_{a}\right)$ is a $\Pi_{n+1}$-sentence, so if $T+\operatorname{REF}^{n}\left(\phi_{a}\right)$ proves a $\Sigma_{n+1}$-sentence $\psi, T$ proves the $\Sigma_{n+1}$-sentence $\operatorname{REF}^{n}\left(\phi_{a}\right) \supset \psi$, which is true by the assumption on $T$, so $\psi$ is true. We will in fact assume that $T_{0}$ is sound, and since our concern is only with the completeness theorem, $T_{0}$ can be taken to be PA, the weakest theory considered.

The above definition of " $n$-reflection sequence" does not in itself tell us anything about how to define $n$-reflection sequences. Such sequences could conceivably be defined in many ways, but if they are to be at all relevant to what we can prove on the basis of iterated reflection, there are some constraints. First, we must be able to prove the two equations above, and preferably in a theory no stronger than PA. Indeed in the case of the first condition, we may reasonably require that if the axioms of a theory $T_{a}$ are defined by a formula $\phi(x)$, the axioms of $T_{\operatorname{suc}(a)}$ should (for $n<\omega$ ) be defined by the formula $\phi(x) \vee x=\bar{\psi}$, where $\psi$ is $\operatorname{REF}^{n}(\phi)$. No similarly concrete formulation suggests itself in the case of limit ordinals, but at least the second equation should be elementarily provable. A second constraint stems from considering that there is no apparent way that we can recognize a sequence of theories $T_{a}$ for $a<_{\mathbf{O}} b$ as sound if we have no uniform method 
for generating the axioms of $T_{a}$ given $a<_{\mathbf{o}} b$. At a minimum, the relation " $a<_{\mathbf{0}} b$ and $\phi$ is an axiom of $T_{a}$ " should thus be effectively enumerable as a relation between a and $\phi$, for any $b$ in $P$. This is in itself a weak condition that does not guarantee that a sequence of theories has any relation to mathematical knowledge, but these two constraints together suggest a natural way of defining reflection sequences, namely as the restriction to a path in $\mathbf{O}$ of a verifiable recursive progression, or just progression for short. A progression based on a theory $T$ is defined as a primitive recursive mapping taking every $a$ in $N$ to a $\Sigma_{1}$-formula $\phi_{a}$, such that PA proves

$$
\begin{aligned}
& T_{0} \text { is } T, \text { and for every } a \text { : } \\
& T_{\text {suc }(a)}=T_{a}+\operatorname{REF}^{n}\left(\phi_{a}\right), \\
& T_{\lim (a)}=\bigcup_{n} T_{\{a\}(n)} .
\end{aligned}
$$

(If $x$ is not 0 or of the form $\operatorname{suc}(a) \operatorname{or} \lim (a), \phi_{x}$ can be given any arbitrary value.) Here the second equation means that for every $n$ such that $\{a\}(n)$ is defined, the axioms of $T_{\{a\}(n)}$ are included among the axioms of $T_{\lim (a)}$.

A progression is not itself a sequence, but for every path $P$ in $\mathbf{O}$ we get an $n$-reflection sequence by restricting the progression to $P$. Further, the existence of progressions follows from the recursion theorem, and therewith the existence of corresponding reflection sequences for every $P$. (For details of how the recursion theorem is used here, see Franzén [2004] or Feferman [1962b].) We will refer to a reflection sequence obtained in this way from a progression as a progressive reflection sequence. Thus every $T_{a}$ for which $a$ is in $\mathbf{O}$ is an iterated extension by reflection of $T_{0}$. If $a$ is not a notation, the corresponding theory $T_{a}$ in a progression has no particular significance, and in particular will not in general be consistent.

The restriction of a progression to the set $\mathbf{O}^{+}$of non-zero notations will be called an ordinal progression. The point of the technicality of having $|a|>0$ for every $T_{a}$ in an ordinal progression will emerge below.

The above definition of a progression is the one given in Feferman [1962b]). The proof of the completeness theorem hits an odd snag when this definition is used, and for this reason a modified definition, due to Beklemishev, will be introduced in Section 5. At this point, however, we will consider that large part of the completeness theorem which does not depend on reflection principles at all.

§4. Shoenfield's completeness theorem. Shoenfield proved in Shoenfield [1959] the completeness of an infinitary rule of inference called the recursive $\omega$-rule, a result used in Feferman's completeness proof, with which it was almost contemporaneous. (Feferman credits Kreisel with having suggested a connection between Feferman's work and Shoenfield's theorem.) 
Feferman's proof in Feferman [1962b] invokes Shoenfield's theorem only after characterizing the theorems of a progression as the closure of the base theory under the recursive $\omega$-rule. We can simplify matters somewhat by formulating Shoenfield's theorem in terms directly applicable to progressions. Beginning with Shoenfield's theorem also serves to make clearer the dividing line between two different ways in which the complete sequences of iterated extensions by reflection yielded by Feferman's theorem leave our actual mathematical knowledge behind, and to bring out more clearly the role played by the $\Pi_{2}$-completeness theorem to be presented in Section 7.

The recursive $\omega$-rule differs from the ordinary $\omega$-rule, by which $\forall x \phi(x)$ follows from the infinitely many premises $\phi(\overline{0}), \phi(\overline{1}), \ldots$, in that proofs using the recursive $\omega$-rule can be represented as finite objects. This does not prevent the rule from being complete, since the resulting finite proofs do not form a recursive (or indeed arithmetically definable) set. A proof of $\phi$ in a system incorporating the recursive $\omega$-rule is either a pair $\langle\phi, 0\rangle$ where $\phi$ is an axiom, or a sequence $\left\langle\phi, e_{1}, \ldots, e_{n}\right\rangle$ where $e_{i}$ is a proof of $\psi_{i}$, and $\phi$ follows from $\psi_{1}, \ldots, \psi_{n}$ by some ordinary inference rule, or, if $\phi$ is $\forall x \psi$, a pair $\langle\phi, e\rangle$, where $e$ is the index of a total recursive function such that $\{e\}(n)$ is a proof of $\psi(\bar{n})$ for every $n$. In the formulation here considered, only ordinary predicate logic derivations are used, but instead we get a set of indexes of theories corresponding to the set of proofs in Shoenfield's formulation.

Shoenfield states in passing at the end of his short paper that the wellorderings defined in Kreisel, Shoenfield, and Wang [1959] can be used to give the bound $\omega^{\omega}$ on the ordinal of a proof, and also that "similar considerations" apply to sentences in second order arithmetic with only universal function quantifiers. The proof of Shoenfield's theorem given below is restricted to proofs of arithmetical sentences and does not require us to consider the rather involved well-orderings of Kreisel, Shoenfield, and Wang [1959]. It uses instead the simple well-founded (but not total) ordering of the "canonical tree" of Mints [1976] associated with an arithmetical statement, which also yields the sharper bound $\omega^{2}$ on the ordinal of a proof tree. (See on this topic also Göran Sundholm's dissertation Sundholm [1983].) ${ }^{1}$

The theorem applies, in the present formulation, to any way of assigning theories $T_{a}$ (assumed to extend PA) to every $a$ in some set $B$ of natural numbers. We will call such an assignment a family of theories. (Thus progressions are a special case of families.) We say that a family is closed under the recursive $\omega$-rule if there is a recursive function $H$ such that for any formula $\phi$ with one free variable $x$ and any total recursive $\{e\}$ with values in $B$, if $T_{\{e\}(n)}$ proves $\phi(\bar{n})$ for every $n, H(e, \phi)$ is in $B$ and $T_{H(e, \phi)}$ proves $\forall x \phi$. We can now state

${ }^{1}$ The proof given here is one suggested by the referee. 
Shoenfield's completeness theorem: For any family of theories which is closed under the recursive $\omega$-rule and any true arithmetical sentence $\phi$, there is a $b$ in $B$ such that $T_{b}$ proves $\phi$.

A natural question is whether the recursive $\omega$-rule like the ordinary $\omega$-rule can prove any true $\Pi_{n}$-sentence when the rule is itself restricted to $\Pi_{n}$ sentences. Shoenfield's original proof left this question open, but we will see that this is indeed the case. We say that a family is $\Pi_{n}$-closed under the recursive $\omega$-rule if the stated condition holds for every $\Pi_{n}$-formula $\forall x \psi$. The proof will establish that the completeness theorem holds for true $\Pi_{n^{-}}$ sentences and families of theories $\Pi_{n}$-closed under the recursive $\omega$-rule.

A family for which $B=\mathbf{O}^{+}$will be called an ordinal family (Turing's "ordinal logics"), and we will also prove the following

Ordinal bound in Shoenfield's theorem: For an ordinal family, if for every $\psi$ and every $e$ with values in $\mathbf{O}^{+},|H(e, \psi)| \leq \sup |\{e\}(n)| * \alpha$, $b$ can be chosen so that $|b|<\alpha^{\omega^{2}}$.

For the proof of the completeness theorem, we will define, given an arithmetical sentence $\phi$, a primitive recursive relation $<_{\phi}$ with the property that $\phi$ is true if and only if $<_{\phi}$ is well-founded. To define $<_{\phi}$, we consider an infinitary system of rules for deriving sequents (finite sequences) $\Gamma=\phi_{1}, \ldots, \phi_{n}$, where each $\phi_{i}$ is an arithmetical sentence in prenex form, using as existential quantifiers $\exists^{m}$, where $\exists^{m} x \phi(x)$ is interpreted as $\exists x(x \geq \bar{m} \wedge \phi(x))$. $\Gamma$ is interpreted as the disjunction of the formulas in the sequence, so that $\Gamma$ is true if and only if at least one of $\phi_{1}, \ldots, \phi_{n}$ is true.

The system has axioms $\phi, \Gamma$ where $\phi$ is a true quantifier-free formula.

There are three rules. The first, writing the premise above the conclusion, is

\section{$\Gamma$}

$\phi, \Gamma$

where $\phi$ is a false quantifier-free formula.

The second rule is the existential quantifier rule,

$$
\begin{gathered}
\Gamma, \phi(m), \exists^{m+1} x \phi(x) \\
\exists^{m} x \phi(x), \Gamma
\end{gathered}
$$

and the final rule, the universal quantifier rule, is the one that makes the system of rules non-finitary:

$$
\begin{aligned}
& \Gamma, \phi(\overline{0}) \quad \Gamma, \phi(\overline{1}) \quad \Gamma, \phi(\overline{2}) \ldots \\
& \forall x \phi(x), \Gamma
\end{aligned}
$$

The leftmost formula in the conclusion of a rule is called the principal formula in the application of the rule, while the other formulas are side formulas. The three rules are not only valid but invertible, that is, the conclusion of a rule is true if and only if every premise is true. 
Now suppose we are given a sentence $\phi$ in prenex form, with every existential quantifier $\exists x$ written $\exists^{0} x$, and with at least one existential quantifier. (We add a dummy existential quantifier if there is none in a sentence to begin with.) We define the canonical tree (CT) for $\phi$ as the tree of sequent numbers obtained by starting from the empty tree, associated with $\phi$, and including, for every $s$ in CT associated with a sequent $\Gamma$ which is not an axiom, the immediate successor or the immediate successors of $s$ corresponding to the premises in the (unique) rule with $\Gamma$ as its conclusion. $<_{\phi}$ is the converse of the standard partial ordering of the sequence numbers, restricted to the sequence numbers in $\mathrm{CT}$.

$<_{\phi}$ is a primitive recursive relation. For this, we need only verify that CT is a primitive recursive set. We verify that the function $F$ defined by $F(s)=$ the sequent associated with $s$ in the above definition of CT, or 0 if $s$ is not in the canonical tree of $\phi$ (where we assume that 0 is not a sequent), is primitive recursive. This is so since $F(\langle\rangle)=\phi$, and $F(s *\langle n\rangle)$ is 0 if $F(s)$ is 0 or the principal formula of $F(s)$ is not a universal formula and $n>0$, or $F(s)$ is an axiom, whereas in the remaining cases $F(s *\langle n\rangle)$ is given in terms of $F(s)$ as indicated in the rules.

To see that $<_{\phi}$ is not well-founded if $\phi$ is false, we note that at least one sequent among the premises of any false sequent in CT is false, and by starting with a false $\phi$ and taking the leftmost false premise at each step we get an infinite path in CT. For the converse, we need to show that if CT has an infinite path $P, \phi$ is false. First we note that if $\Gamma$ is a sequent in $P$, every side formula $\psi$ in $\Gamma$ appears as the principal formula in a later sequent in $P$, because of the rotation built into the rules. (Formally this follows by induction on the number of formulas preceding $\psi$ in $\Gamma$.) We can now prove by induction on the complexity (number of quantifiers) of a formula $\psi$ in $\Gamma$ in $P$ that every such $\psi$ is false. If $\psi$ is quantifier-free, $\psi$ is false, since otherwise $P$ would contain an axiom. If $\psi$ is $\forall x \phi(x)$, some instance $\phi(\bar{n})$ will occur in a sequent in $P$, and is false by the induction hypothesis, so $\psi$ is false. If $\psi$ is $\exists^{m} x \phi(x), \phi(\bar{k})$ will appear in sequents in $P$ for every $k \geq m$, since every $\exists^{k} x \phi(x)$ will be the principal formula at some point, so $\psi$ is false.

For a sequent $\Gamma$, let $\Gamma^{*}$ be the disjunction of the formulas in $\Gamma$, and let $\Gamma_{s}$ denote the sequent associated with $s$ in CT. To prove the completeness theorem, we define, using the recursion theorem, a primitive recursive function $G$ such that for every $s$ in CT,

$$
\begin{aligned}
& \text { if } \Gamma_{s} \text { is an axiom, } G(s)=0, \\
& \text { if } \Gamma_{s} \text { is the conclusion of a 1-premise rule, } G(s)=G(s *\langle 0\rangle), \\
& \text { if } \Gamma_{s} \text { is } \forall x \phi(x), \Gamma, G(s)=H\left(e, \forall x\left(\phi(x) \vee \Gamma^{*}\right)\right) \\
& \text { where }\{e\}(m)=G(s *\langle m\rangle) .
\end{aligned}
$$

Assuming $\phi$ to be true, we can now prove by $<_{\phi}$-induction that $T_{G(s)}$ proves $\Gamma_{s}^{*}$ for every $s$ in CT. In particular, $T_{G(\langle\rangle)}$ proves $\phi$. The proof is straightfor- 
ward, using the fact that every true quantifier-free sentence is provable in PA and the logical equivalence of $\forall x\left(\phi(x) \vee \Gamma^{*}\right)$ and $\forall x \phi(x) \vee \Gamma^{*}$, given that $\Gamma^{*}$ contains no free variables.

Now assume $\phi$ is a true $\Pi_{n}$-sentence. In defining the canonical tree, we assumed a dummy existential quantifier to be added (in order to ensure that the canonical tree of a false sentence is not well-founded), but even with such a dummy existential quantifier included, we find that every sentence in every sequent $\Gamma$ in the canonical tree of $\phi$ is equivalent in PA to a $\Pi_{n}$-sentence. $\Pi_{n}$-closure under the recursive $\omega$-rule is therefore sufficient for every true $\Pi_{n}$-sentence to be provable.

The ordinal bound. What is the ordinal of the canonical tree for a true formula $\phi$ ? We define the canonical tree for a sequent $\Gamma$ like the canonical tree for a formula $\phi$, except that we start from $\Gamma$ instead of $\phi$. We prove by induction on $n$ that the ordinal of the canonical tree beginning with $\Gamma$ is strictly smaller than $\omega *(n+1)$ if $\Gamma$ contains a true sentence of complexity $n$. For this we need the following observation (proved by an easy induction on $m$ ): if every path of a tree leads after at most $m$ steps to a node of order $\leq \alpha$, the tree has ordinal at most $\alpha+m$. Now suppose $\Gamma$ contains a true formula of complexity 0 . Then, because of the strict rotation of formulas as principal formula, there is an $m$ such that every path will lead to an axiom in at most $m$ steps. If $\Gamma$ contains a true sentence $\forall x \phi(x)$ of complexity $n$, after at most $m$ steps every path will lead to an application of the universal quantifier rule to a sequent $\forall x \phi(x), \Gamma^{\prime}$, and by the induction hypothesis the ordinal of that sequent is at most the supremum of $\omega *(n-1)+k_{1}+1$, $\omega *(n-1)+k_{2}+1 \ldots$ for some numbers $k_{1}, k_{2}, \ldots$, and so the ordinal of $\Gamma$ is at most $\omega * n+m$. The case when $\Gamma$ contains a true existential sentence is treated similarly.

This allows us to obtain the upper bound for $|b|$ in Shoenfield's completeness theorem when $B=\mathbf{O}^{+}$. Suppose $|H(e, \phi)| \leq \sup |\{e\}(n)| * \alpha$ for every $e$. We then get, for the infinitary rule, that $|G(s)| \leq \sup |G(n)| * \alpha$ with the supremum taken over all $n$ such that $n<_{\phi} s$. An inductive argument then yields that $|G(s)| \leq \alpha^{\operatorname{ord}(s)}$ for every $s$, and using the bound given above we get $|b|=|G(\langle\rangle)|<\alpha^{\omega^{2}}$.

Shoenfield's completeness theorem and mathematical knowledge. For a trivial example of a family of sound theories which is closed under the recursive $\omega$-rule, let $B$ be the set of true arithmetical sentences, and define $T_{\phi}$ as the theory obtained from PA by adding $\phi$ as a new axiom. Here we do not need Shoenfield's theorem to conclude that every true $\phi$ is provable in $T_{\phi}$. In spite of being utterly trivial, this example is typical of the irrelevance of the completeness theorem to mathematical knowledge. Given $\phi$, we know that $\phi$ if true is provable in a sound theory constructed from $\phi$, but we know that theory to be sound only if we know $\phi$ to be true. Similarly with ordinal progressions: given $\phi$, we know that $\phi$ if true is provable in a theory $T_{b}$ con- 
structed from $\phi$, but we know $T_{b}$ to be a theory in the ordinal progression (or equivalently, know $b$ to be in $\mathbf{O}$ ), and thereby an iterated extension by reflection of PA, only if we know $\phi$ to be true

For a non-trivial example, if we define $T_{0}$ as PA and again let $T_{\phi}$ be the theory obtained from PA by adding $\phi$ as a new axiom, we can define $B$ inductively by

0 is in $B$,

if $\{e\}$ is total and for every $n,\{e\}(n)$ is in $B$ and $T_{\{e\}(n)}$ proves $\phi(\bar{n})$, $\forall x \phi(x)$ is in $B$.

$T_{b}$ is sound for every $b$ in $B$, and by the completeness theorem the resulting family of theories proves every true arithmetical sentence. The set $B$ corresponds to the set of derivations of the form $\langle\phi, e\rangle$ in Shoenfield's presentation.

Additive families and complete sequences. We say that an ordinal family is additive if $T_{a+b}$ extends both $T_{a}$ and $T_{b}$. Given an additive family of theories closed under the recursive $\omega$-rule, we can define a sequence of theories such that every true arithmetical sentence is provable in some theory in the sequence. Starting from an enumeration $\phi_{1}, \phi_{2}, \ldots$ of all true arithmetical sentences, we choose $b_{i}$ as any $b$ such that $T_{b}$ proves $\phi_{i}$, and then let $a_{k}=b_{1}+\cdots+b_{k}$. Defining $B$ as the set of notations a such that $a<_{\mathbf{0}} a_{k}$ for some $k$, we get a path. Restricting the family to $B$ yields a sequence of theories in which every $\phi_{i}$ is provable. The length of this sequence, on the same assumption as in the statement of the ordinal bound in Shoenfield's theorem, will be bounded by $\alpha^{\omega^{2}} * \omega$.

We can make a further observation concerning additive families. We say that a family of theories is locally closed under the recursive $\omega$-rule if there is a recursive $G$ such that for any formula $\phi$ with one free variable $x$ and any $a$ in $B$, if $T_{a}$ proves $\phi(\bar{n})$ for every $n, G(a, \phi)$ is in $B$ and $T_{G(a, \phi)}$ proves $\forall x \phi$. If an additive family of theories is locally closed under the recursive $\omega$-rule with a $G$ such that $|G(a, \phi)| \leq|a| * \alpha$, it is closed under the recursive $\omega$-rule with an $H$ such that $|H(e, \phi)| \leq \sup |\{e\}(n)| * \omega * \alpha$. This is so because if $T_{\{e\}(n)}$ proves $\phi(\bar{n})$ for every $n, T_{\lim (a)}$ proves $\phi(\bar{n})$ for every $n$, where $\{a\}(k)=\{e\}(0)+\cdots+\{e\}(k)$, so taking $H(e, \phi)$ to be $G(a, \phi)$ we get $|H(e, \phi)| \leq \sup |\{e\}(n)| * \omega * \alpha .(\lim (a)$ is a notation since $|\{e\}(n)|$ is never 0$)$. If $\alpha \leq \omega^{m}$ for some finite $m$, we get the bound $\left(\omega^{m}\right)^{\omega^{2}} * \omega=\omega^{\omega^{2}+1}$ for the sequence defined above. This yields what we may call the

Reflection-independent part of Feferman's completeness theorem: Given an additive family of theories which is locally closed under the recursive $\omega$-rule with $|G(a, \phi)| \leq|a| * \omega^{m}$ for some finite $m$, a sequence of theories of length $\omega^{\omega^{2}+1}$ can be extracted such that every true arithmetical sentence is provable in some theory in the sequence. 
The full completeness theorem results from showing that an ordinal progression is an additive family of theories satisfying the stated condition.

Local closure under the recursive $\omega$-rule can also be restricted to $\Pi_{n}$ sentences, and we get a corresponding version of the reflection-independent part of Feferman's completeness theorem for true $\Pi_{n}$-sentences.

§5. Smooth progressions. Ordinal progressions, as defined in Section 3, have a major drawback: they are not additive. Or rather, there is no apparent way of showing them to be additive, even though no counterexample seems to be known. Feferman comments on this difficulty in Feferman [1962b] and gets around the problem by less than satisfactory means. (A more complicated property corresponding to additivity is proved using the $\Pi_{2}$ completeness theorem.)

Consider any progression, and an $a$ in $\mathbf{O}$. The mapping that takes $b$ to $T_{b}^{\prime}=T_{a+b}$ is then also a progression, based on $T_{a}$. We thus have two progressions, one based on $T_{0}$ and another based on $T_{a}$. Since $T_{a}$ extends $T_{0}, T_{b}^{\prime}=T_{a+b}$, and $T_{b}=T_{0+b}$, one would expect $T_{b}^{\prime}$ to extend $T_{b}$ for $b$ in $\mathbf{O}$. Since $a<_{\mathbf{O}} a+b$ for $a$ and $b$ in $\mathbf{O}, T_{a+b}$ extends $T_{a}$, so this would mean that ordinal progressions are indeed additive. But although no immediate counterexample suggests itself, there is no apparent way of showing that $T_{b}^{\prime}$ extends $T_{b}$ (at least not outside the autonomous part of a progression - a distinction which does not have any role to play in the present paper).

A solution of this difficulty is found in Beklemishev [1995] notion of a smooth progression. The axioms introduced in a reflection sequence are all reflection principles for earlier theories in the sequence, and by using this property in characterizing progressions we can do away with the troublesome limit ordinal case for the purpose of establishing additivity.

A smooth progression is accordingly defined as a primitive recursive mapping taking every $a$ in $N$ to a $\Sigma_{1}$-formula $\phi_{a}$ such that PA proves

For every $a$, the axioms of $T_{a}$ are the axioms of $T_{0}$ together with all $\operatorname{REF}^{n}\left(\phi_{b}\right)$ for $b<_{\mathbf{K}} a$.

As before, we get a notion of ordinal progressions and of progressive $n$ reflection sequences, the difference now being that we avoid the difficulties associated with the earlier definition. Indeed it holds for smooth progressions that PA proves that for any two progressions, if $T_{0}$ extends $T_{0}^{\prime}, T_{a}$ extends $T_{a}^{\prime}$ for every $a$. However, it is no longer the case that the mapping taking $b$ to $T_{a+b}$ is a (smooth) progression, or at least this is not provable in any obvious way. We therefore instead prove directly that PA proves that for every $a$ and $b$, $T_{a+b}$ extends $T_{b}$. The proof (adapting the proof in Beklemishev [1995], uses Löb's theorem: if PA proves "if PA proves $\phi$ then $\phi$ ", then PA proves $\phi$. (This kind of use of Löb's theorem was introduced by Schmerl in his method of "reflexive induction".) 
Some preliminaries are needed. First, it will turn out that the proof needs

$$
\text { if } a<_{\mathbf{K}} b \text { and } b \text { is not } \operatorname{suc}(a) \text { then } \operatorname{suc}(a)<_{\mathbf{K}} b
$$

This does not follow from the (standard) definition of $<_{\mathbf{K}}$ given earlier, so we just add the above as a further clause in the inductive definition of $<_{\mathbf{K}}$. It still holds that $<_{\mathbf{K}}$ is an effectively enumerable relation that coincides with $<_{\mathbf{O}}$ on $\mathbf{O}$.

Second, we need to observe that PA proves that $a+b<_{\mathbf{K}} a+c$ whenever $b<_{\mathbf{K}} c$. Finally, we need monotonicity: using the definition of a progression, PA proves that if $a<_{\mathbf{K}} b$ then $T_{b}$ extends $T_{a}$.

So we need to prove in PA, on the assumption that PA proves "for every $a$ and $b, T_{a+b}$ extends $T_{b}$ ", that for every $a$ and $b, T_{a+b}$ extends $T_{b}$. Rather than complicate the notation, we give an informally worded proof which inspection shows to be formalizable in PA.

We need to show that every axiom of $T_{b}$ is provable in $T_{a+b}$. An axiom $\psi$ of $T_{b}$ is either an axiom of $T_{0}$, in which case it is also an axiom of $T_{a+b}$, or is $\operatorname{REF}^{n}\left(\phi_{d}\right)$ for some $d<_{\mathbf{K}} b$. Since PA proves that $T_{a+d}$ extends $T_{d}$, $\operatorname{REF}^{n}\left(\phi_{d}\right)$ is provable in $T_{\text {suc }(a+d)}$. Further $\operatorname{suc}(a+d)=a+\operatorname{suc}(d)$, and since $d<_{\mathbf{K}} b$ we get $\operatorname{suc}(d) \leq_{\mathbf{K}} b$, so $\operatorname{suc}(a+d) \leq_{\mathbf{K}} a+b$, which by monotonicity implies that $T_{a+b}$ proves $\psi$.

In the remainder of this paper, by a progression will be meant a smooth progression. Using smooth progressions greatly simplifies the later parts of Feferman's proof.

We now come to the part of Feferman's proof that depends essentially on the use of carefully formulated reflection principles, which is the proof that an $\omega$-reflection progression is locally closed under the recursive $\omega$-rule. To see this idea at work in a much simpler case, we first look at Turing's earlier completeness result.

§6. Turing's completeness theorem. Turing proved a completeness theorem for $\Pi_{1}$-sentences, which in the present framework becomes

Turing's completeness theorem: For any true $\Pi_{1}$-sentence $\phi$ and any consistency progression there is an $a$ in $\mathbf{O}$ with $|a|=\omega+1$, given as a primitive recursive function of $\phi$, such that $T_{a}$ proves $\phi$.

Thus in terms of sequences, there is a consistency sequence

$$
T=T_{0}, T_{1}, \ldots, T_{\omega}, T_{\omega+1}
$$

such that $\phi$ is provable in $T_{\omega+1}$. This is on the face of it an interesting result, and one naturally wonders just how the proof of $\phi$ in $T_{\omega+1}$ makes use of the infinitely many consistency statements that are axioms of $T_{\omega+1}$. The answer, as the proof of the theorem shows, is that the axioms of $T_{0}, T_{1}, \ldots, T_{\omega}$ are irrelevant to the theorem.

For the proof, let $\phi=\forall x \psi(x)$ with $\psi$ in $\Delta_{0}$. We define $e$ so that provably in PA 
For every $n$,

$$
\begin{array}{ll}
\{e\}(n)=n_{\mathbf{O}} & \text { if } \forall x<n \psi(x), \\
\{e\}(n)=\operatorname{suc}(\lim (e)) & \text { otherwise. }
\end{array}
$$

If $\phi$ is true, $\lim (e)$ is in fact a notation for $\omega$, and $T_{\operatorname{suc}(\lim (e))}$ proves $\forall x \psi(x)$, because provably in PA, if $\phi$ is false, $T_{\lim (e)}$ proves its own consistency, and is therefore inconsistent.

Considering how the axioms of $T=T_{0}, T_{1}, \ldots, T_{\omega}$, are defined in $T_{\omega+1}$, if we start from a canonical definition of the axioms of $T$, we see that the axioms of $T_{i}$ for $i<\omega$ are given canonical definitions, whereas the axioms of $T_{\omega}$ are given a non-standard definition corresponding to the definition of $e$. The reason why a non-standard description appears only at $\omega$ is that in a progressive reflection sequence, it is only at limit ordinals and at 0 that a non-standard definition of the axioms of a theory can be introduced. If we start instead from a non-standard definition of the axioms of $T$, we can simplify the construction in the proof correspondingly, obtaining a

Non-progressive version of Turing's completeness theorem: For any

$T$ and any true $\Pi_{1}$-sentence $\phi$, there is a $\Sigma_{1}$-definition $\theta$ of the axioms of $T$ yielding a consistency extension $T+\operatorname{Con}(\theta)$ of $T$ in which $\phi$ is provable.

The proof of this is simple. If $\phi$ is $\forall x \psi$ and $\phi_{1}$ is any $\Sigma_{1}$-formula with free variable $y$ defining the axioms of $T$, take $\theta$ to be the formula

$$
\phi_{1} \vee(\exists x \neg \psi \wedge y=\perp) \text {. }
$$

Here $\perp$ can be taken to stand for the formula $0=s(0)$. For a version closer to Turing's construction, if $\phi_{1}$ is a primitive recursive definition of the axioms of $T$, we get another primitive recursive definition by

$$
\left(\phi_{1} \wedge \forall x \leq y \psi\right) \vee(\exists x \leq y \neg \psi \wedge y \text { is any formula }) .
$$

We thus see that the essential point in Turing's completeness proof is the use of a non-standard definition of the axioms of $T$ in formulating " $T$ is consistent". The appearance of an infinity of theories in the statement of the theorem is due to the constraints on a progressive reflection sequence. These constraints make good sense when we are talking about what we can (actually or potentially) prove on the basis of reflection, but in the completeness theorem they merely obscure what is going on in the argument. These features partly carry over to Feferman's far-reaching extension of Turing's argument, which will be considered next.

§7. Feferman's $\Pi_{2}$-completeness theorem. The key to proving that an $\omega$ progression is closed under the recursive $\omega$-rule is the following

$\Pi_{2}$-completeness theorem: For any $n$-reflection progression, where $n>0$, there is a recursive $F$ such that for any true $\Pi_{2}$-sentence $\psi$, $a=F(\psi)$ is in $\mathbf{O},|a|=\omega^{2}+\omega+1$ and $T_{a}$ proves $\psi$. 
The term $\omega^{2}$ appears in the formulation of the result again because it is only at limit ordinals that new non-standard definitions of the axioms of a theory can be introduced in a progression. As in the case of Turing's completeness theorem, there is a simplified

Non-progressive version of the $\Pi_{2}$-completeness theorem: For any true $\Pi_{2}$-sentence $\psi$ and base theory $T$ there is a 1-reflection sequence of length $\omega+1$ in which the last theory proves $\psi$.

The proof of the non-progressive version is in this case not at all trivial, but is essentially the same as Feferman's proof. Since it makes the essential idea clearer, we here give (in somewhat compressed form) a proof of the nonprogressive version rather than of Feferman's formulation. (An exposition of Feferman's original proof is given in Franzén [2004].)

It is conceivable that there is, as in the case of Turing's completeness theorem, a 1-extension sequence of length 1 rather than $\omega+1$ in which $\psi$ is provable. (The 1-reflection principle for $T$ is itself a $\Pi_{2}$-sentence.) When you think about it, there is however no obvious way of formulating any such immediate version of the $\Pi_{2}$-completeness theorem, and Feferman's construction uses an infinity of theories in an essential way.

The overall approach of the argument can be described as follows. We will define a recursive function $H$ taking any theory $T$ (given by a $\Sigma_{1}$-formula) and true $\Sigma_{1}$-sentence $\phi$ to an extension $H(T, \phi)$ of $T$ by finitely iterated 1 -reflection in which $\phi$ is provable. Given a true $\Pi_{2}$-sentence $\forall x \exists y \psi(x, y)$ we then define

$$
\begin{aligned}
T_{0} & =T, \\
T_{n+1} & =H\left(T_{n}, \exists y \psi(\bar{n}, y)\right) .
\end{aligned}
$$

For the union $T_{\omega}$ of these theories, which is an $\omega$ times iterated 1-reflection extension of $T$, it follows that

$$
\text { For every } n, \exists y \psi(\bar{n}, y) \text { is provable in } T_{\omega} .
$$

If we can now find a 1-reflection extension $T_{\omega+1}$ of $T_{\omega}$ in which (1) is provable, it follows that $T_{\omega+1}$ proves $\forall x \exists y \psi(x, y)$. On first inspection, this may look like a singularly unpromising approach. (1) is itself a $\Pi_{2}$-sentence, and why should it be any easier to find an extension by 1-reflection in which (1) is provable than to find one in which $\forall x \exists y \psi(x, y)$ is provable? Also, by $\Sigma_{1}$-completeness, every instance $\exists y \psi(\bar{n}, y)$ is provable in PA, so the above construction applies if we take $H(T, \phi)$ to be $T$, which gets us nowhere.

The key to the practicality of the above approach lies in making the construction independent of the assumption that $\forall x \exists y \psi(x, y)$ is true. Recall Turing's construction: given any $\Pi_{1}$-sentence, we construct an extension $T_{1}$ of $T$ in which we can show that sentence to be provable, whether or not it is true. If it is true, $T_{1}$ is in fact a consistency extension of $T$. Similarly in Feferman's proof, (1) will hold whether or not $\forall x \exists y \psi(x, y)$ is true, and $\phi$ is 
always provable in $H(T, \phi)$. If $\phi$ is true, $H(T, \phi)$ is in fact an extension by finitely iterated 1-reflection of $T$, so if $\forall x \exists y \psi(x, y)$ is true, $T_{\omega}$ is an $\omega$ times iterated extension by 1 -reflection of $T$.

The way the construction of $H(T, \phi)$ works is by defining a descending sequence of extensions by iterated 1-reflection. A theory $H(T, \phi)=T_{0}^{*}$ is defined as an extension by reflection of a theory $T_{1}^{*}$, which in turn is defined as an extension by reflection of $T_{2}^{*}$, and so on. If $\phi$ is provable in $T$, this sequence will lead to some $k$ for which $T_{k}^{*}$ and every later theory is $T$, and by reversing the sequence we get a finite sequence of iterated extensions by 1-reflection of $T$. However, if $\phi$ is not provable in $T$, the sequence will be an infinitely descending one, and every theory in this infinitely descending sequence proves $\phi$. In some cases the theories in the sequence will be inconsistent, while in other cases they will be consistent although proving the false sentence $\phi$. The theories $T_{k}^{*}$ will be defined by specializing the parameter $w$ in a double sequence of theories $T_{w, k}$ to a value $d$ chosen through a devious application of the recursion theorem. The theories $T_{w, k}$ use non-standard definitions incorporating Turing's construction, and as in the case of the non-progressive version of Turing's completeness theorem, the absence of an intervening $\omega$-sequence of standardly defined theories between $T_{w, k}$ and $T_{w, k+1}$ accounts for the length of the sequence being $\omega$ rather than (as in Feferman's original proof) $\omega^{2}$.

Now for the details. We will prove that $\phi$ is provable in $H(T, \phi)$, for any theory $T$ and $\Sigma_{1}$-sentence $\phi$. The proof can be formalized in a standard extension by 1-reflection of PA: we will define $H$ so that it is provable in PA that there is, for every $\phi$ and $T$, a proof in PA that $\phi$ is provable in $H(T, \phi)$. Note the indirection in the argument: it does not show it to be provable in PA that $\phi$ is provable in $H(T, \phi)$ for every $\phi$ and $T$, but rather it is provable in PA that a certain primitive recursive function yields, for every $T$ and $\phi$, a proof in PA that $\phi$ is provable in $H(T, \phi)$.

To obtain $H$, we define, using the recursion theorem and the simplified Turing construction, a sequence of theories $T_{w, k}$ as a primitive recursive function of $T, \phi, w, k$ (where $T$ extends PA and $\phi$ is a $\Sigma_{1}$-sentence) so that PA proves (where $\{w\}^{2}$ is the two-place partial recursive function with index $w$ ):

If there is a proof smaller than $k$ in $T$ of $\phi$, then $T_{w, k}$ is $T$. (Here and in the following, "proof" means "PR-proof".)

Otherwise $T_{w, k}$ extends $T$ by the 1-reflection principle for $T_{w, k+1}$, and furthermore, if there is an $n$ for which $\{w\}^{2}(k+1, n)$ is defined and is not a proof in PA that $\phi$ is provable in $T_{w, k+1}, T_{w, k}$ also has the axiom $0=s(0)$.

Again, as in the use of the recursion theorem to define progressions, what is actually defined in PA is a primitive recursive function taking as values certain $\Sigma_{1}$-formulas defining the axioms of $T_{w, k}$. The axioms of $T$ are defined using the same formula throughout. What is needed for the argument, as 
will be seen, is that it is provable in PA that for every consistent $T_{w, k}$, there is a proof in PA that $\phi$ is provable in $T_{w, k+1}$, and it is in order to ensure this by means of Turing's construction that the formulation "every $\{w\}^{2}(k+1, n)$ is a proof in PA that $\phi$ is provable in $T_{w, k+1}$ " is used. Thus using the extra parameter $w$ makes it possible to replace an existential statement "There is a proof in PA of ..." by a $\Pi_{1}$-statement "Every value of $w$ is a proof in PA of ...".

For the proof, we note that there is a primitive recursive $G$ such that if $r$ is a proof in PA that $\{w\}^{2}$ is total, $G(r, w, \phi, k)$ is a proof in PA that $\phi$ is provable in $T_{w, k}$. This is the proof formalizing the following argument: If there is a proof smaller than $k$ in $T$ of $\phi$, then $T_{w, k}$ is $T$, and so $\phi$ is provable in $T_{w, k}$. Otherwise, if $T_{w, k}$ is inconsistent, anything is provable in $T_{w, k}$. If $T_{w, k}$ is consistent, every $\{w\}^{2}(k+1, n)$ is either undefined or a proof in PA that $\phi$ is provable in $T_{w, k+1}$, and since $\{w\}^{2}$ is total (here the proof $r$ is used), there is such a proof in PA, and hence in $T_{w, k}$. Since $T_{w, k}$ proves 1-reflection for $T_{w, k+1}$, it follows that $\phi$ is provable in $T_{w, k}$. (As a technical comment which will not be pursued in this paper, the argument actually only requires $T_{w, k}$ to prove what is known as the local principle of $\Sigma_{1}$-reflection for $T_{w, k+1}$.)

The final step is a somewhat unusual application of the recursion theorem. We define $d$ as a primitive recursive function of $\phi$ and $T$ so that for every $\phi$ and $T$,

$$
\{d\}^{2}(k, n)=G(r, d, \phi, k) \text {, where } r \text { is a proof in PA that }\{d\}^{2} \text { is total. }
$$

(Such a $d$ can be defined because the proof in PA that the function defined by the primitive recursion theorem is total does not use any particulars about the primitive recursive functions involved - see Franzén [2004] for details.) Thus for every $n,\{d\}^{2}(k, n)$ is a proof in PA that $\phi$ is provable in $T_{d, k}$. In particular, for every $\phi$ and $T,\{d\}^{2}(0,0)$ is a proof in PA that $\phi$ is provable in $T_{d, 0}$, and so for every $\phi$ and $T$, there is a proof in PA that $\phi$ is provable in $H(T, \phi)=T_{d, 0}$. Furthermore, if $\phi$ is in fact provable in $T, H(T)$ is a finite 1-reflection extension of $T$.

The argument by which $\forall x \exists y \psi(x, y)$ is provable in $T_{\omega+1}$ can now be carried through, taking $T_{\omega+1}$ to be an extension by 1-reflection of $T_{\omega}$, where the axioms of $T_{\omega}$ are defined as above. ( $T_{\omega+1}$ proves (1) provided that the axioms of $T$ are defined in such a way that PA and thereby $T$ proves that every theorem of PA is a theorem of $T$.)

The definition of the axioms of $T_{\omega}$ used in $T_{\omega+1}$ in proving $\forall x \exists y \psi(x, y)$ is on inspection seen to be non-standard to say the least. We define the theory as the union of a certain effectively given sequence of theories, where the theories in the sequence are themselves defined in convoluted terms in such a way that if $\forall x \exists y \psi(x, y)$ is true each theory is a finite iterated extension by 1 -reflection of the preceding theory, although this can in no way be read off from the definition. 
Let us compare the $\Pi_{2}$-completeness theorem, the proof of which is highly non-trivial and involves a striking use of the recursion theorem, with another " $\Pi_{2}$-completeness theorem", which is trivial: for any true $\Pi_{2}$-sentence $\phi$, there is an axiomatization of $T$ such that the 1-reflection principle for $T$ using this axiomatization implies $\phi$ in PA. For, if $\phi$ is true, we get an equivalent axiomatization of $T$ by including as an axiom $\exists y \psi(\bar{n}, y)$ for every $n$ (by $\Sigma_{1}$-completeness), and using this axiomatization, 1-reflection immediately implies $\phi$.

As far as relevance to mathematical knowledge is concerned, there is not much to choose between these two $\Pi_{2}$-completeness theorems. In one case we use an axiomatization of a theory which we know to be an axiomatization of the theory only if we know $\phi$ to be true, in the other we use a description of the axioms of a theory which we know to be a description of the axioms of the theory only if we know $\phi$ to be true. But the proof of Feferman's $\Pi_{2}$ completeness theorem is clearly an interesting one, unlike the proof of the trivial $\Pi_{2}$-completeness theorem. It is a natural feeling that Feferman's result is the more significant one, but the significance of the result is not to be sought in any relation to mathematical knowledge. Just where that significance lies is not a question that will be answered in this paper, but we will instead go on to see how $\Pi_{2}$-completeness leads to the full completeness theorem. One other application should be mentioned: Feferman used the argument in his paper Feferman [1962a] on hierarchies of recursive functions, which solved a problem posed by Kleene.

$\S 8$. Feferman's full completeness theorem. The $\Pi_{2}$-completeness theorem implies that $\omega$-reflection progressions are locally closed under the recursive $\omega$-rule. For suppose $T_{b}$ proves $\phi(\bar{n})$ for every $n$. Then it is true that for every $n, \phi(\bar{n})$ has a PR-proof in $T_{b}$, and so there is, by the $\Pi_{2}$-completeness theorem, an $a$ given by a recursive function such that $T_{a}$ proves "for every $n$, $\phi(\bar{n})$ is provable in $T_{b}$ ". $T_{\operatorname{suc}(b)+a}$ therefore proves $\forall x \phi$, since $T_{\operatorname{suc}(b)}$ proves $\omega$-reflection for $T_{b}$. Further,

$$
|\operatorname{suc}(b)+a|=|b|+1+\omega^{2}+\omega+1<|b|+\omega^{3} \leq|b| * \omega^{3}
$$

since $|b|>0$. By the argument in Section 4 , this implies

Feferman's completeness theorem: Given an $\omega$-reflection progression, an $\omega$-reflection sequence of length $\omega^{\omega^{2}+1}$ can be extracted such that every true arithmetical sentence is provable in some theory in the sequence.

Feferman formulated his theorem with the bound $\omega^{\omega^{\omega}+1}$ instead of $\omega^{\omega^{2}+1}$, since he based his use of Shoenfield's completeness theorem on Shoenfield's paper, which gave the bound $\omega^{\omega}$ for the depth of applications of the recursive $\omega$-rule. The improved bound thus does not involve any modification of Feferman's reasoning. The main simplification results from using additive ordinal progressions. 
The proof that $\omega$-reflection progressions are locally closed under the recursive $\omega$-rule also shows that for $n>0, n$-reflection progressions are locally $\Pi_{n+1}$-closed under the recursive $\omega$-rule. The relativized version of Shoenfield's completeness theorem thus yields a

Relativized Feferman's completeness theorem: For $n>0$, given an $n$-reflection progression, an $n$-reflection sequence of length $\omega^{\omega *(n+1)+1}$ can be extracted such that every true $\Pi_{n+1}$-sentence is provable in some theory in the sequence.

(A similar theorem was claimed in Fenstad [1968], but was invalidated by the error in that paper.)

It would be misleading to say that the completeness theorem shows that we, or an idealized mathematician, will "eventually" obtain every arithmetical truth by iterating reflection principles, since completeness depends essentially on a very careful choice of path in the set $\mathbf{O}$ of ordinal notations. (Indeed in Feferman and Spector [1962] it is shown that there are paths through $\mathbf{O}$ - paths giving a notation to every constructive ordinal - such that the corresponding sequences derived from any of a wide category of families of theories do not even prove every true $\Pi_{1}$-sentence.) It may also be a bit misleading to speak of a "choice of path" here, suggesting as it does that by intuitively sniffing out the right choice at each fork, we could prove any true arithmetical sentence. "Making the right choice" in the sense of the theorem is in fact equivalent to choosing (at limit ordinals) a particularly convoluted definition of the axioms of a theory in the sequence, a definition which we know to actually define those axioms only if we already know the sentence which we seek to prove to be true. If we consider how the full completeness theorem follows from local closure under the recursive $\omega$-rule, we see that iteration of the construction in the proof of the $\Pi_{2}$-completeness theorem is used to obtain ever more convoluted non-standard definitions of the axioms of theories.

§9. Concluding comments. The technical notion of a progressive $n$ reflection sequence yields a general framework for the study of what can be known or proved on the basis of iterated arithmetical reflection principles. In particular, the concept of an autonomous sequence is useful in considering what can be proved in iterated extensions by reflection that we (at least potentially) recognize as such. Another branch of this study, in which autonomy plays no role, leads to Feferman's completeness theorem, which shows that there are relatively short progressive $\omega$-reflection sequences in which every true arithmetical sentence is provable. The completeness theorem can be seen as a dramatic illustration of the role of intensionality in logic. In the case of PA and ZF and other theories that we actually work with in logic, there is a clear distinction between standard and non-standard definitions 
of their axioms, and therewith between standard and non-standard formulations of reflection principles. But when we pose the general question just what can be proved on the basis of reflection starting from some unspecified theory $T$, we have no such distinction between standard and non-standard formulations of reflection principles, and must say that this depends on how the axioms of the theory are defined - not just on what those axioms are. This indeed was a starting point for Feferman's work in Feferman [1960]: Gödel's second incompleteness theorem is commonly said to show that the consistency of a theory $T$ cannot be proved in $T$ itself, but for this to be true we must impose a suitable condition on the formulation of " $T$ is consistent". It turned out that for the second incompleteness theorem to hold, a sufficient condition is that " $T$ is consistent" is formalized using a $\Sigma_{1}$-definition of the axioms of the theory in a straightforward formalization of "no contradiction is derivable using the rules of predicate logic from the axioms of $T$ ". This condition is not however sufficient to rule out formalizations of " $T$ is consistent" with irrelevant mathematical implications, as is most simply shown by Turing's completeness theorem. Thus we can easily define $\omega$-reflection sequences based on PA of length $\omega^{\omega^{2}+1}$ which we recognize as such, but we can also by using non-standard definitions show the existence of such sequences which prove every true arithmetical sentence, although for the theories $T_{a}$ in this sequence, we have no basis for claiming that we know or could know the truth of " $T_{a}$ is an iterated $\omega$-reflection extension of PA". We are thus faced with the very general question which theories have canonical axiomatizations, and how such axiomatizations can be systematized.

Acknowledgments. I thank Grigori Mints and Göran Sundholm for helpful pointers. Particular thanks to the referee for useful suggestions, and for a better proof of Shoenfield's completeness theorem.

\section{REFERENCES}

L. BeKLemishev [1995], Iterated local reflection versus iterated consistency, Annals of Pure and Applied Logic, vol. 75, pp. 25-48.

S. FEFERMAN [1960], Arithmetization of metamathematics in a general setting, Fundamenta Mathematicae, vol. 49, pp. 35-92.

S. FefERman [1962a], Classifications of recursive functions by means of hierarchies, Transactions of the American Mathematical Society, vol. 104, pp. 101-122.

S. FEFERMAN [1962b], Transfinite recursive progressions of axiomatic theories, The Journal of Symbolic Logic, vol. 27, pp. 259-316.

S. Feferman And C. Spector [1962], Incompleteness along paths in progressions of theories, The Journal of Symbolic Logic, vol. 27, pp. 383-390.

J. FENSTAD [1968], On the completeness of some transfinite recursive progressions of axiomatic theories, The Journal of Symbolic Logic, vol. 33, pp. 69-76.

T. FRANZÉn [2004], Inexhaustibility: a non-exhaustive study, Lecture Notes in Logic, Association for Symbolic Logic and A K Peters, To appear.

R.O. Gandy and C.E.M. Yates (editors) [2001], Mathematical logic, Collected Works of A. M. Turing, Elsevier, Amsterdam. 
P. HÁJEK AND P. PUdLÁK [1993], Metamathematics of first-order arithmetic, SpringerVerlag, Berlin, New York.

G. KReISEL, J. SHOENFIELD, AND H. WANG [1959], Number theoretic concepts and recursive well-orderings, Archiv fur Mathematische Logik und Grundlagenforschung, vol. 5, pp. 42-64.

M. H. Löв [1955], Solution of a problem of Leon Henkin, The Journal of Symbolic Logic, vol. 20, pp. 115-118.

G. MinTs [1976], The universality of the canonical tree, Soviet Mathematical Doklady, vol. 17 , no. 2 .

U. R. SCHMERL [1979], A fine structure generated by reflection formulas over primitive recursive arithmetic, Logic colloquium '78 (M. Boffa, D. van Dalen, and K. McAloon, editors), vol. 97, North-Holland, Amsterdam, pp. 335-350.

J. R. Shoenfield [1959], On a restricted $\omega$-rule, L'Académie Polonaise des Sciences. Bulletin. Série des Sciences Mathématiques, Astronomiques et Physiques, vol. 7, pp. 405-407.

J. R. Shoenfield [1967], Mathematical logic, Addison-Wesley Publishing Co., Reading, MA., reprinted by the Association for Symbolic Logic, 2001.

G. Sundholm [1983], Proof theory: A survey of the omega rule, Oxford D. Phil. dissertation.

DEPARTMENT OF COMPUTER SCIENCE AND ELECTRICAL ENGINEERING

LULEA UNIVERSITY OF TECHNOLOGY

SE-97187 LULEÅ, SWEDEN

E-mail: torkel@sm.ltu.se 